\title{
GENERATION OF SHORT X-RAY PULSES USING CRAB CAVITIES AT THE ADVANCED PHOTON SOURCE*
}

\author{
K. Harkay\#, M. Borland, Y.-C. Chae, G. Decker, R. Dejus, L. Emery, W. Guo, D. Horan, K.-J. Kim, \\ R. Kustom, D. Mills, S. Milton, A. Nassiri, G. Pile, V. Sajaev, S. Shastri, G. Waldschmidt, M. White, \\ B. Yang, ANL, Argonne, IL 60439, U.S.A. \\ A. Zholents, LBNL, Berkeley, CA U.S.A.
}

\begin{abstract}
There is growing interest within the user community to utilize the pulsed nature of synchrotron radiation from storage ring sources. Conventional third-generation light sources can provide pulses on the order of 100 ps but typically cannot provide pulses of about $1 \mathrm{ps}$ that some users now require to advance their research programs. However, it was recently proposed by A. Zholents et al. to use rf orbit deflection to generate sub-ps X-ray pulses [1]. In this scheme, two crab cavities are used to deliver a longitudinally dependent vertical kick to the beam, thus exciting longitudinally correlated vertical motion of the electrons. This makes it possible to spatially separate the radiation coming from different longitudinal parts of the beam. An optical slit can then be used to slice out a short part of the radiation pulse, or an asymmetrically cut crystal can be used to compress the radiation in time. In this paper we present a feasibility study of this method applied to the Advanced Photon Source (APS). We find that the pulse length can be decreased down to a 1 ps range using superconducting crab cavities.
\end{abstract}

\section{INTRODUCTION}

Storage-ring-based synchrotron radiation sources produce light in pulses with a typical duration of several tens to hundreds of ps, defined by the equilibrium electron bunch length. Although this pulse length is adequate for many applications, new science horizons could be opened if high-brightness light sources with shorter pulses were available [2]. In the future, linac-based free-electron lasers (FELs) [3] or energy-recovery linacs (ERLs) [4] may produce pulse lengths in the $100 \mathrm{fs}$ range or less. However, there is a broad class of dynamic phenomena that occur in the 1-100 ps range. We propose a method to produce high-brightness $\mathrm{x}$-ray pulses that could be implemented at APS to improve the temporal resolution from the current 100 ps level to $\sim 1$ ps full width half maximum (FWHM).

The approach relies on manipulating the transverse momentum of the electron bunch using deflection. Radiation from the deflected particle beam can be either sliced or combined with time-compression $\mathrm{x}$-ray optics, resulting in a beam with a pulse duration of $\sim 1$ ps. In the case of compression, the time-averaged flux is in excess of $10^{13} \mathrm{ph} / \mathrm{s}$ (at $10 \mathrm{keV}$ ), amounting to a reduction to $\sim 1 / 10$ of the normal flux at this energy. In continuous wave (cw) mode, the short-pulse repetition rate can be as high as the

\footnotetext{
*Work supported by U.S. Department of Energy, Office of Basic Energy Sciences, under Contract No. W-31-109-ENG-38.

"harkay@aps.anl.gov
}

rf frequency of the APS ring (352 MHz), depending on the bunch spacing. While femtoslicing [5] gives shorter $\mathrm{x}$ ray pulses, this approach gives significantly higher flux at a much higher repetition rate. Such a source would be unique in the world and would be complementary to future x-ray FELs, combining high repetition rate with all the advantages of access, stability, and tunability of synchrotron light sources.

Driven by the considerable scientific interest generated at a recent APS workshop [2], a study was undertaken to analyze the feasibility of applying the rf deflection technique for pulse shortening at APS. A major criterion was to minimize the impact of such a scheme on the existing APS performance, i.e., low emittance and high photon brightness for all other users not using the short pulses. Several challenges emerged in the study, but we believe that none of these are showstoppers.

\section{THE CONCEPT}

The approach we are considering for short-pulse production is based on a concept developed by Zholents et al. [1]. Deflection is introduced by installing two rf cavities operating in the $\mathrm{TM}_{110}$ mode in the ring; these are also known as "crab" cavities and have been studied for use in high-luminosity particle colliders [6]. A horizontal magnetic field creates a correlation between the longitudinal coordinate of electrons within the bunch and their vertical angles, producing a "chirped" beam (Fig. 1). The cavity frequency is a harmonic $h$ of the ring $r f$ frequency. The second crab cavity cancels the deflection, thereby limiting the deflection to a part of the ring. The required deflection can be achieved with $\mathrm{h}=4-8(1.4-2.8 \mathrm{GHz})$ and deflecting voltages of 4-6 MV. With such parameters, superconducting $\mathrm{rf}$ (SRF) technology is required for $\mathrm{cw}$ operation.

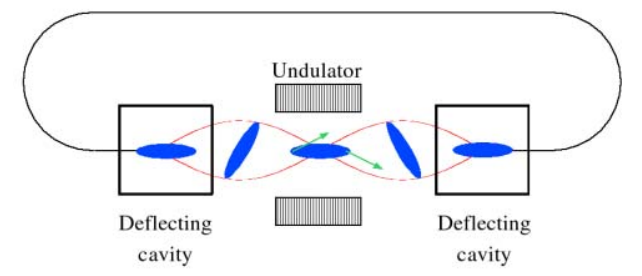

Figure 1: Schematic of the rf deflection approach to producing short pulses.

When the chirped bunch passes through an undulator, the radiation pulse that is emitted becomes correlated in vertical position and time as it propagates (Fig. 2). Short pulses can be extracted from the chirped pulse by simply installing a slit that limits the vertical extent of the beam 
that is passed to the sample. A more efficient approach, which uses a larger vertical portion of the beam, uses time-compressing $\mathrm{x}$-ray optics. Compression can improve the throughput by up to an order of magnitude compared to slitting alone for a fixed pulse length.

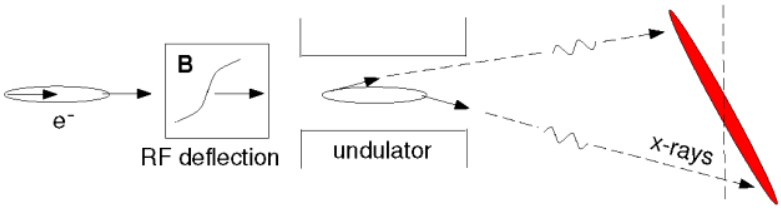

Figure 2: The chirped pulse can be sliced or compressed with an asymmetric cut crystal.

Feasibility issues in the areas of beam dynamics, SRF system, and compression optics are discussed below. It should be noted that other accelerator-based techniques have been proposed for generating short, transient radiation pulses $[7,8]$, but these have low repetition rates and other considerations and are not discussed further here.

\section{BEAM DYNAMICS}

An extensive study of the impact of the crab cavities on the beam dynamics in the APS was performed [9]. Pulses of 1 ps require $h \geq 4$, and good lifetime requires that $V \leq 6$ MV. For the APS, $2.5 \mathrm{~nm}$ emittance, $1 \%$ coupling, $100 \mathrm{ps}$ bunch length (FWHM) (for 24 bunches at $100 \mathrm{~mA}$ ), and $9.6 \times 10^{-4}$ energy spread were assumed. One of the major findings was significant vertical emittance growth due to large vertical trajectories in the sextupoles between the cavities. Simply turning off the internal sextupoles leads to other problems. First, the lifetime is degraded because the sextupole distribution symmetry is broken. Second, the uncompensated chromaticity leads to emittance growth due to the energy spread.

Sextupole optimization was performed to compensate the emittance growth while correcting the chromaticity to zero between the cavities [10]. For $h=8$ and $4 \mathrm{MV}$ or less, after optimization the vertical emittance growth is smaller than a factor of two, and we can maintain the normal vertical beam size by adjusting the initial coupling (to $0.3 \%$ at $4 \mathrm{MV}$ ). The lifetime was estimated by calculating the dynamic aperture. For the optimized sextupole case, the lifetime is expected to be better than with sextupoles turned off, but less than with normal sextupoles.

Assuming linear deflection, the compressed x-ray pulse length is given by $\sigma_{t, x r a y}=\frac{E}{2 \pi h f_{0} V} \sqrt{\sigma_{y^{\prime}, e}^{2}+\sigma_{y^{\prime}, \text { rad }}^{2}}$, where $E$ is the beam energy ( $7 \mathrm{GeV}$ for the APS), $V$ is the deflecting voltage, $f_{0}$ is the ring $\mathrm{rf}$ frequency, $\sigma_{y^{\prime}, e} \approx 2.0$ $\mu \mathrm{rad}$ is the electron beam divergence, and $\sigma_{y^{\prime}, \text { rad }} \approx 5 \mu \mathrm{rad}$ is the zero-emittance undulator radiation divergence. For example, for $\mathrm{h}=8$ and $4 \mathrm{MV}$, the calculated $\mathrm{x}$-ray pulse length is $\sim 0.5 \mathrm{ps}$ rms.

More realistic simulations of the photon distributions using a simple compression model were also carried out $[9,10]$. Sample results are given in Fig. 3 , where $\Delta t_{70}$ is about twice the rms value. The pulse length is under $2 \mathrm{ps}$ with $60-80 \%$ transmission over a range of $\mathrm{h}$ (optics transmission is not included).

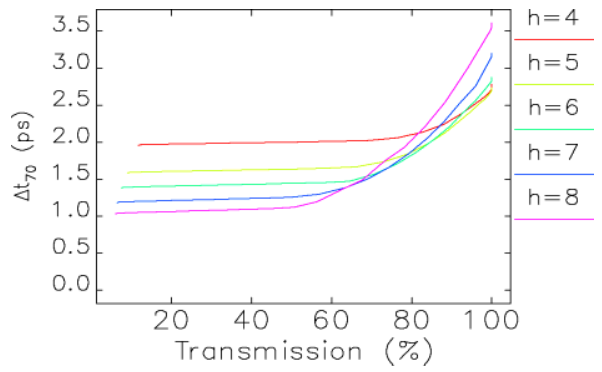

Figure 3: Compression results for $10 \mathrm{keV}$ radiation, assuming APS undulator A and $4 \mathrm{MV}$ deflecting voltage.

The effects of errors were also studied in detail [9]. The most challenging tolerance is on the $\mathrm{rf}$ phase error. Phase errors can result in vertical emittance growth, as well as centroid motion outside the crabbing region. The cavityto-cavity phase error should be less than 0.04 degrees to keep the effects to within $10 \%$. The voltage difference should be less than $0.5 \%$.

Finally, a preliminary evaluation was made of impedance effects. The vacuum chamber vertical impedance is not expected to degrade the chirp performance. The coupled-bunch instability thresholds due to the SRF lower order (LOM) and higher order (HOM) modes were also estimated. Preliminary results at $100 \mathrm{~mA}$ give stability limits of $R_{s} \times f<0.8 \mathrm{M} \Omega-G H z$ and $R_{t}<2.5 \mathrm{M} \Omega / m$, where $R_{s}$ is the longitudinal shunt impedance, $f$ is the mode frequency in $\mathrm{GHz}$, and $\mathrm{R}_{\mathrm{t}}$ is the transverse impedance.

\section{SRF CRAB CAVITIES}

A preliminary design of the SRF system was carried out. Design constraints include available space $(2 \times 2.5 \mathrm{~m})$, availability of $100 \mathrm{~kW}$ class cw rf amplifiers, and LOM/ HOM damping requirements. For the baseline, $h=8$ and 4 MV were chosen. The system would operate at $2 \mathrm{~K}$ to reduce BCS losses. The unloaded quality factor is $3 \times 10^{9}$. A squashed-cell shape was used to remove the $\mathrm{TM}_{110}$ degeneracy, modeled after the KEK design [6]. To keep surface fields within reasonable limits (surface magnetic field $\leq 100 \mathrm{mT}$ ), either seven single-cell or three 4-cell cavities are required to give $4 \mathrm{MV}$. Single-cell cavities are in principle easier to damp, while 4-cell cavities are more compact. The LOM/HOM damping design is to be studied in more detail, after which the cryomodule and cavity configuration will be determined. Very recently, we also began to consider a normal-conducting pulsed rf option.

\section{TIME-COMPRESSION X-RAY OPTICS}

The time-compression optical system presented interesting technical challenges due to the unusual parameters of the x-ray beam ( $500 \mu \mathrm{rad}$ vertical divergence), which required somewhat unorthodox optical designs to be considered. Criteria included maximizing the compression throughput, minimizing the pulse length and spot size, and providing energy-scanning tunability. 
The compression optical element is an asymmetrically cut crystal (or crystals). In such a crystal, the surface is not parallel to the reflecting crystallographic planes, and the spatial tilt angle of an x-ray pulse rotates upon reflection. A suitable geometry can produce an outgoing tilt that is perpendicular to the propagation direction, representing a temporally compressed pulse. In addition to simulations of the undulator radiation following rf deflection, x-ray optics compression simulations were conducted exploring a variety of schemes: asymmetric crystals in both Bragg (reflection) and Laue (transmission) geometries, flat and bent crystals, and with and without transversely focusing mirrors [11].

Using as an example with no optics compression, for $\mathrm{h}=8$ and 4 MV deflecting voltage, a vertical aperture of $600 \mu \mathrm{m}$ transmits $\sim 2 \%$ of the x-rays and result in a $1.8 \mathrm{ps}$ FWHM pulse. Some x-ray studies could benefit without any compression (sliced only), while still attaining ps time resolution: e.g., x-ray transmission spectroscopy. An example of using optics to compress a larger part of the chirped x-ray pulse is shown in Fig. 4 (slit aperture 24 $\mathrm{mm})$. The first crystal of a $\mathrm{Si}(400)$ double-crystal monochromator is cut with a $-14.5 \mathrm{deg}$. asymmetry angle in order to eliminate the $-46 \mathrm{deg}$. tilt of the incident x-rays. This expands the $20 \mathrm{~mm}$ beam to $70 \mathrm{~mm}$. Vertical focusing is accomplished by a horizontally reflecting mirror with a sagittally curved profile. A similar collimating mirror is placed before the first crystal to avoid a large energy spread (see Fig. 4). The time distribution of the radiation in the final beam is 1.9 ps FWHM (Fig. 5). Taking into account the bandwidths of the crystals and the reflectivities of the crystals and mirrors, the flux in the final spot is about an order of magnitude more than that obtained by slitting alone to achieve the same pulse width.

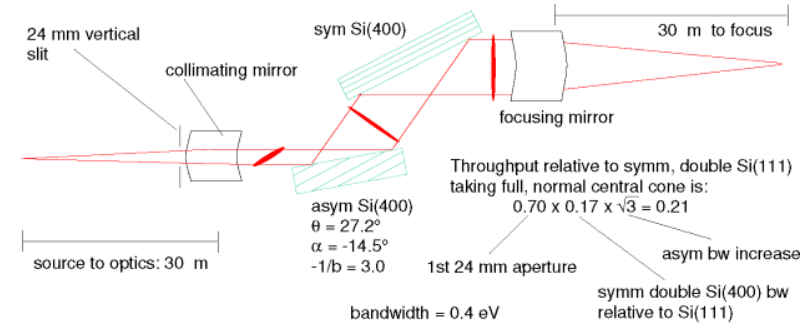

Figure 4: An optics example to compress (to $1.9 \mathrm{ps)}$ and focus $10 \mathrm{keV}$ x-rays ( $\mathrm{h}=8,4 \mathrm{MV}$ ).

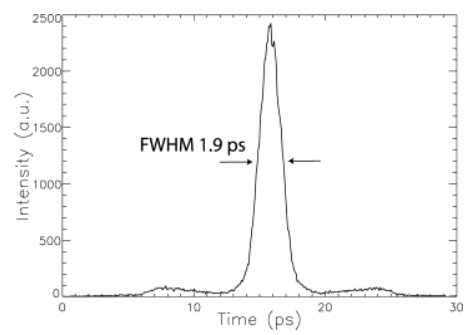

Figure 5: Pulse histogram of the first harmonic at $10 \mathrm{keV}$ using the geometry in Fig. 4. The transmission is $62 \%$ (optics reduction factor not applied).

Optimization simulations of the x-ray compression optics have been conducted over a large parameter space. To cover the energy range between $5-40 \mathrm{keV}$, no more than three tunable monochromators are required. The throughput enhancement of the compression optics over slitting alone varies between 15 to 2 -fold over $5-30 \mathrm{keV}$ and becomes no better than slitting at around $40 \mathrm{keV}$. The ability to scan energy while preserving the pulse-compression condition requires a "variable asymmetry" on one of the crystals, which can be accomplished by azimuthally rotating the crystal.

\section{IMPLEMENTATION AT THE APS}

Current simulations indicate that the two rf cavities can be installed up to four straight sections apart. A twosector implementation is shown schematically in Fig. 6. In this configuration, three insertion devices (IDs) (or four, if the IDs in the middle sector were canted) on consecutive sectors would have the short-pulse capability.

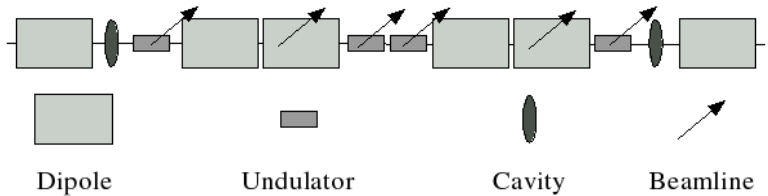

Figure 6: Schematic of a two-sector implementation.

\section{SUMMARY}

We find that a short-pulse capability can be implemented in the APS. Such an upgrade would overcome the current $100 \mathrm{ps}$ barrier, providing a unique capability to perform time-resolved x-ray experiments with $\sim 1$ ps pulses. This capability is complementary to the even shorter pulses expected from the x-ray FELs. Implementation would involve installing SRF crab cavities across 2-4 sectors and upgrading existing ID beamlines to provide both sliced and compressed x-ray pulses.

\section{REFERENCES}

[1] A. Zholents, P. Heimann, M. Zolotorev, J. Byrd, Nucl. Instrum. Methods, A425, 385 (1999).

[2] Future Scientific Directions for APS (http://www.aps.anl.gov/ Future/Strategic_Planning_Meeting/home.htm); 2005 APS Users Meeting: Workshop on Generation and Use of Short X-ray Pulses at APS (http://www.aps.anl.gov/Users/Meeting/)

[3] LCLS Design Study Report, SLAC-R-521, 1998; TTF-FEL Conceptual Design Report, TESLA-FEL 95-03, DESY, June 1995; J. Rossbach et al., Nucl. Instrum. Methods A375, 269 (1996).

[4] J.B. Murphy, Proc. of the 2003 PAC, 176 (2003).

[5] A. A. Zholents, M. S. Zolotorev, Phys. Rev. Letts. 76, 912 (1996), and R. W. Schoenlein et al., Science 287, 2237 (2000).

[6] K. Akai, Y. Morita, "Crab Cavity for High-Current Accelerators," KEK-Preprint-2003-123 (2003).

[7] W. Guo, K. Harkay, B. Yang, M. Borland, V. Sajaev, "Generating Picosecond X-ray Pulses with Beam Manipulation in Synchrotron Light Sources," these proceedings.

[8] G. Decker, N. Sereno, "Transient Generation of Short Pulses in the APS Storage Ring," these proceedings.

[9] M. Borland, submitted to PRST-AB.

[10] M. Borland, V. Sajaev, "Simulations of X-ray Slicing and Compression Using Crab Cavities in the Advanced Photon Source," these proceedings.

[11] S.D. Shastri, R.J. Dejus, L. Assoufid, private communication. 